\title{
Application of RFID Technology in Design of E-Commerce Supply Chain System In Internet of Things
}

\author{
Haoyuan $\mathrm{Ou}^{1}{ }^{1}$ a $^{*}$, Jianming Zhang ${ }^{1}$ and Yi Wang ${ }^{1}$ \\ ${ }^{1}$ Department of Information Engineer, Guangdong Polytechnic, Foshan, China \\ adongleiyuedu@163.com
}

\begin{abstract}
Keywords: Supply chain system; Logistics management; Warehouse and inventory management; RFID; Internet of things
\end{abstract}

\begin{abstract}
The application of RFID technology in the supply chain is to facilitate communication between suppliers and customers. Successful logistics management can seamlessly integrate all supply activities and integrate all participants into the supply chain. The key of modern logistics management is the automatic identification of products, containers, vehicles and personnel in the supply chain. The application of supply chain management system based on RFID technology can fundamentally solve the problem of inventory and supply chain management. The paper presents application of RFID technology in design of e-commerce supply chain system in Internet of things.
\end{abstract}

\section{Introduction}

Now if the RFID system with the existing barcode system are combined, which can effectively solve the problem of the flow of goods and warehouse and information related to the management, not only can increase the number of processing goods within one day, all the flow of information can also view these goods. The combination of bar code and RFID technology is also a way of RFID application in this stage. The utility model can be used for sticking the bar code on the articles, the radio frequency electronic label is pasted on the tray or the forklift for storing articles, and the electronic label is used for storing the information of all the articles on the tray or the forklift.

RFID may eventually replace the ubiquitous bar code and become the mainstream technology in the field of logistics management in the future. Compared with bar code technology and the widely used electronic label, in addition to save the manual operation, also has the waterproof, antimagnetic, high temperature resistance, long service life, reading distance and other advantages. In addition, because the data on the electronic label can be encrypted, the storage capacity of the data is large, and the storage information can be changed, therefore, the utility model has wider application range than the bar code and is more convenient to use. The popularization and application of RFID will bring revolutionary changes to the retail and logistics industries. RFID technology has many advantages, so it has a wide range of applications.

Because RFID carries the electronic information, its data content can be protected by password, so that it's content is not easy to be forged and altered. In recent years, RFID has attracted much attention because of its characteristics such as remote reading, high storage capacity and so on. It can not only help an enterprise to significantly improve the efficiency of goods, information management, can also make the sales and manufacturing enterprises interconnection, and thus more accurately receive feedback information, control demand information, optimize the supply chain.

The Internet is extended on the basis of the Internet and the extension of communication standards and protocols to a certain basis, through the information sensing device, information exchange and communication, realize the person to person $(\mathrm{H} 2 \mathrm{H})$, to $(\mathrm{h} 2 \mathrm{t})$ and $(\mathrm{T} 2 \mathrm{~T})$ to interconnect, its core is to realize the interconnection between objects the realization of information exchange and communication between objects. The application in the field of logistics network, this is called "the third profit source" of the development of the industry, domestic science workers have done a lot of basic research, has a very important significance for the further development of the Internet of things 
logistics. The paper presents application of RFID technology in design of e-commerce supply chain system in Internet of things.

\section{Development and Analysis of Warehouse Logistics Management Based on RFID Tags}

Today's bar code can not be changed after printing; RFID tags stored in the data can be updated dynamically, can be recycled and used repeatedly. No matter penetration and screen reading, barcode scanner must be in close and no objects blocking the case, can read the bar code. RFID can penetrate the paper, wood and plastic and other non-metallic and non transparent material, conducting communication, no light source, read farther. However, it is not recognized by conductive objects such as metal.

Real time storage system, update the warehouse and inventory management and product positioning, temporary permanent check - can be executed immediately when receiving delivery mixed pallets can save time and improve accuracy to reduce delivery cost. In the warehouse guidance and tracking with RFID tag forklift - real time localization, optimize asset utilization, better measure of output monitoring change in stock inventory accuracy rate close to $100 \%$, reduce inventory number, simplify procedures to promote sales potential, as is shown by equation (1).

$$
\begin{aligned}
\bar{R}(m) & :=\operatorname{diag}\{\bar{R}(m, 1), \bar{R}(m, 2), \cdots, \bar{R}(m, M)\} \\
& :=\operatorname{diag}\{R((m-1) M+1), R((m-1) M+2), \cdots, R(m M)\}
\end{aligned}
$$

Goods distribution center is mainly warehouse, it plays all the information collection, distribution, record of goods, can be said that the warehouse is an important part of the logistics, it always appears in the combination of the logistics, such as procurement and production, production processing and finishing, the conversion between different the mode of transportation etc.. Accurate and efficient storage is the premise and foundation of the whole logistics supply chain efficiency improvement, how to improve the accuracy and efficiency of warehousing is the urgent problem to be solved.

When the container is closed, the sensor starts to collect the temperature and humidity regularly, at the same time monitor whether the container door is opened illegally, and whether it is hit or not. When the container is in and out of the checkpoint, the label records the time and place. When there is a need for the reader, the tag must be able to respond in time and provide relevant information to the reader.

Using the RFID technology, you can track the goods in the supply system in real time, indicating the specific location of a commodity at a certain time, and reduce the loss of goods in the inventory. RFID technology has been successfully applied in some stores, especially for those with high profit or expensive goods. The formula used in the method is as follows.

$$
E\left\{x_{i}\right\}=\left\{\begin{array}{c}
E\left\{x_{1 t}\right\} \\
\vdots \\
E\left\{x_{n t}\right\}
\end{array}\right\}=\left\{\begin{array}{c}
\mu_{1 t} \\
\vdots \\
\mu_{n t}
\end{array}\right\}
$$

Logistics often pull more than and 10 people come, and even vendors also sent several people, a rummage, huge manpower and material resources. And, in order to facilitate the search for goods, you need to pile up the goods to stay in the channel, so down, warehouse utilization rate of only $30 \%$. Customers and logistics are not satisfied with this. In this case and it is how to solve the problem of improving the efficiency and the utilization rate of warehouse. The emergence of RFID has become a life-saving straw to solve logistics problems.

Inaccurate material, BOM errors, lack of effective means to report and communicate problems, poor coordination, no information system support. With the application of warehouse management system based on RFID (radio frequency identification) technology, the problem of inventory management can be solved fundamentally. RFID technology not only eliminates the manual intervention in the process of tracking, but also saves a lot of manpower and greatly improves the 
work efficiency. This system can greatly simplify the inventory management of goods to meet the increasing demand for information flow and information processing speed.

How to create dynamic RFID tag postal industry information platform, installed at the entrance of a reader / in the distribution center, without manual operation, and can meet the requirements of a forklift goods into the warehouse operation / mobile information scanning, and remote dynamic one-time identification of multiple tags. This saves the operation time of storage / stars will be anxious to express queries, improve working efficiency.

\section{Application of RFID Technology in Logistics Management of Intelligent E-Commerce}

The RFID reader controls the transmission of data streams by receiving additional information. Once the reader information is properly received and decoded, the reader through a specific algorithm to decide whether to send the signal transmitter to serve again, or know the stop signal transmitter, which is "command response protocol". The use of such an agreement, even in a very short time, a small space to read a number of labels, but also can effectively prevent the generation of "cheating".

The photo on the surface of the chip can only show the connection of the top metal, which is opaque. With the help of imaging system with high performance, can identify the lower layer information from the top of the rugged, but for the CMOS process provides oxidation layer flattening, you need to remove the metal to further understand the various structures of the under layer. As a result, the CMOS process for the planarization of oxide layers is more suitable for smart card processing including RFID.

Radio frequency identification technology provides a fast, accurate and automatic method for tracking, managing and monitoring the goods. Radio frequency identification technology as the core of the container automatic identification, and it is to become the world's largest cargo tracking application. Warehousing, distribution and other logistics links, radio frequency identification technology in warehousing, distribution and other logistics has been a lot of successful applications. With the research and development of radio frequency identification technology in the open logistics link, the logistics industry will become the biggest benefit industry, as is shown by equation (3).

$$
\left\{\begin{array}{l}
w_{j, \min }^{\xi}(m, n)=\frac{1}{2}-\frac{1}{2}\left[\frac{1-M_{j, A B}^{\xi}(m, n)}{1-T}\right] \\
w_{j, \text { max }}^{\xi}(m, n)=1-w_{j, \text { min }}^{\xi}(m, n)
\end{array}\right]
$$

The use of electronic tags in the electronic label system as an important carrier of electronic information of container in the container, the container number, manufacturer and owner of the detailed information such as input electronic tags, when labeled boxes through the browser, the label will transfer product data to the reader, the reader and then download the data to a central processor, generating container database management.

In this way, the container from the factory to the final discharge all can be clearly understand and grasp, so as to make the container circulation more smooth, can help the departments to know the recycling container dynamic, timely contact with the owner or agent or lessee, contact can be in the form of fax or network, and avoid wasted due to long time unattended, effectively improve the utilization of container, as is shown by equation (4).

$$
G_{t}=\frac{1}{M} \sum_{j=1}^{M}\left(A_{j}-\bar{A}\right)^{T}\left(A_{j}-\bar{A}\right)
$$

Warehouse general business procedures including warehousing contract signed, acceptance of goods, procedures for warehousing, storage of goods, delivery of goods, warehousing management content, ordering, delivery, inventory, delivery inspection, warehouse storage, handling, site management, stock operation etc.. The combination of products in the warehouse, proper stowage and 
circulation packaging, group and other activities is to improve the efficiency of loading and unloading, make full use of transport, thereby reducing the cost of transport costs.

The role of road signs in the entire system is to activate the container tag that wakes up in its neighborhood, providing accurate container location information to the reader. Its structure is similar to that of a reader, including a microprocessor, an antenna drive chip and an interface unit. But the sign only downlink communication, that is, from the road signs to the radio frequency label communication and label free to sign up communication. The road sign is fixed, and each landmark has its own coordinates in the container yard. When it wakes up the label, the data packet contains its own coordinate information, which is forwarded by the label.

The typical application of RFID in the logistics supply chain management is from raw materials to end users all the physical flow process, which includes supplier selection, material procurement, product design, material processing, order processing, inventory management, packaging and transportation, warehouse management and customer service, including products in the supply chain, the owner, location time and other information. The application of RFID technology in the supply chain is to facilitate communication between suppliers and customers. Successful logistics management can seamlessly integrate all supply activities and integrate all participants into the supply chain. According to the different functions of the organization, the participants include suppliers, distributors, carriers, third party logistics companies and information providers.

\section{Application of RFID Technology in Design of E-Commerce Supply Chain System in Internet of Things}

In the logistics RFID system, the biggest "secret" lies in the electronic label on the tray. The length of about $8 \mathrm{~cm}$, width of about $2 \mathrm{~cm}$ of electronic tags, such as a small memory, can store about 1000 bytes. The electronic tag users will be installed on the tray, the goods in storage before, only hand-held barcode information reader reads the pallets of goods, and then into the electronic tag, all information will be stored in the cargo tray to the terminal of the computer, which is fixed in the reader. By virtue of handheld reader, the information before the entry into the work, you can be in the thousands of miles away from the production workshop completed.

Warehouse, warehouse operator according is to the order list check every item, after passing the inspection to the warehouse keeper into the warehouse. The warehouse keeper to the handset has been scanned receiving operation of the storage application code, wireless mobile devices from the warehouse management system to download the scanned storage application material receiving information, material information including: material code, as is shown by equation(5), where material name is quantity of goods received and the tray bar code, and specify whether the material required inspection and inspection if completed, at the same time identifying material whether it is urgent.

$$
\begin{aligned}
C_{j+1, k, m}=\sum_{l, n} \bar{h}_{k-2 l} & \bar{h}_{m-2 n} C_{j, l, n}+\sum_{l, n} \bar{h}_{k-2 l} \bar{g}_{m-2 n} D_{j, l, n}^{1} \\
& +\sum_{l, n} \bar{g}_{k-2 l} \bar{h}_{m-2 n} D_{j, l, n}^{2}+\sum_{l, n} \bar{g}_{k-2 l} \bar{g}_{m-2 n} D_{j, l, n}^{3}
\end{aligned}
$$

Frequency doubled radio frequency identification system to achieve a certain degree of difficulty. In general, the reader sends out a radio frequency signal, and the frequency of the signal returned by the electronic tag is the frequency doubling of the reader. This mode provides the convenience of the reader, receiving and processing the echo signal but for passive electronic tags, electronic tag reader RF energy conversion for receiving echo frequency carrier, its low energy conversion efficiency, improve the micro wave technique requires a high conversion efficiency, which means the higher cost of electronic tags.

RFID technology is closely linked with the logistics supply chain; it is expected to replace the barcode scanning technology in the next few years. The key of modern logistics management is the 
automatic identification of the products, containers, vehicles and personnel in the supply chain. Some information needs to be transmitted and reflected in the enterprise MIS system or ERP system in real time. RFID technology can do this, so it is more common in the field of logistics management.

RFID, as a kind of information technology, its basic function is to achieve accurate and fast data acquisition. After the collection of these data, it is necessary to analyze and deal with the data, in order to improve the efficiency and reduce the cost. In other words, the implementation of RFID, often need to reach a certain level of enterprise information, so that the RFID system and the existing ERP, CRM and other information integration, in order to give full play to its role.

\section{Summary}

The paper presents application of RFID technology in design of e-commerce supply chain system in Internet of things. Modern supply chain management in the use of modern science and technology is the main use of bar code, POS technology. Bar code is by far the most economical and practical automatic identification technology. It has advantages of high speed, high reliability, collecting a large amount of information, flexible and practical, and bar code label is easy to make, no special requirements for equipment and materials, equipment identification of easy operation, no need of special training and equipment are relatively cheap.

\section{Acknowledgements}

This paper is supported by University Innovation Center Science and technology innovation team project of -Guangdong Polytechnic (KJCX201604).

\section{References}

[1] Xiangwei Mu, Yan Chen, Yan Cao, Yan Li, "Personalized Recommendation System Modeling in Semantic Web", AISS, Vol. 5, No. 2, pp. $278 \sim 284,2013$.

[2] He Jialiang, Ouyang Dantong, Wang Haiyan, "A RFID Authentication Protocol with Index Inner Table Used in Reader", JDCTA, Vol. 6, No. 2, pp. $119 \sim$ 127, 2012.

[3] [3] Mohd Helmy Abd Wahab, Norzilawati Abdullah, Ayob Johari, Herdawatie Abdul Kadir, GSM Based Electrical Control System for Smart Home Application, JCIT, Vol. 5, No. 1, pp. 33 39, 2010.

[4] Jinbiao Hou, Design and Implementation of the Temperature Measurement and Control System

[5] Based on the SCM, JCIT, Vol. 8, No. 6, pp. $1219 \sim 1228,2013$. [5] Xiaojun WU, "The Application of Neural Network Technology in Electronic Commerce Evaluation System", JDCTA, Vol. 5, No. 12, pp. $233 \sim 239,2011$.

[6] XianFeng Yang, Zhen Liu, The Application of Association Rules Mining in Building Intelligent Transportation Systems, JCIT, Vol. 7, No. 20, pp. $575 \sim 582,2012$.

[7] Feng Feng, Xu Qi, "Locating Method in the Warehouse Based on Active RFID Tags", JDCTA, Vol. 7, No. 1, pp. $223 \sim 231,2013$.

[8] Hongxin Wan, Yun Peng, "Clustering and Evaluation on Electronic Commerce Customers Based on Fuzzy Set", IJACT, Vol. 5, No. 3, pp. $199 \sim 206,2013$.

[9] Yun-Peng Cai, "Construction Personalized Recommendation System Of E-Commerce Based On Variable Precision Rough Set", IJACT, Vol. 4, No. 11, pp. 125 131, 2012. 